Präv Gesundheitsf 2020 · 15:326-331

https://doi.org/10.1007/s11553-020-00773-1

Eingegangen: 14. Januar 2020

Angenommen: 23. März 2020

Online publiziert: 14. April 2020

(c) Der/die Autor(en) 2020

\author{
Fabian Kerwagen ${ }^{1,2}$ \\ ${ }^{1}$ Münster, Deutschland \\ ${ }^{2}$ Hochschule Fulda, Fulda, Deutschland
}

\title{
Disstressassoziierte Erkrankungen im Arztberuf - Wie wirksam sind resilienzfördernde Interventionen?
}

Eustress (positiver Stress), welcher uns zu hohen Leistungen befähigen kann und des Disstress (negativer Stress), welcher beispielsweise mit Magengeschwüren oder psychischen Erkrankungen assoziiert ist.

In der Literatur werden hohe Prävalenzen dieser disstressbedingten Folgeerkrankungen wie Depression, Burnout oder Suchtverhalten unter Ärztinnen und Ärzten vielfach beschrieben [2, 3, 13]. Dass Disstress im Arztberuf in Deutschland ein relevantes Problem ist, wurde 2013 in einer anonym durchgeführten Online-Umfrage unter 12.062 Klinikärztinnen und -ärzten in Baden-Württemberg nachgewiesen: $55,5 \%$ der Teilnehmer/-innen zeigten Anzeichen von Disstress. Ein Großteil des Krankenhauspersonals klagte über stressbedingte Beschwerden wie Nervosität (64\%), Alpträume (44,1\%), Kopfschmerzen (40,8\%), Schlaflosigkeit (35\%) und gastrointestinale Irritationen (41,4\%) [15] Darüber hinaus besteht bei Ärztinnen und Ärzten eine im Vergleich zur Normalbevölkerung um den Faktor 1,4 (Männer) bzw. 2,3 (Frauen) erhöhte Suizidrate [9].

In der Folge ergeben sich für die Public-Health-, Versorgungsforschungsaber auch Gesundheitsökonomieperspektiven verschiedene Konsequenzen. Erstens wird der bereits vorherrschende Ärztemangel verschärft [4]. Zweitens ist ärztlicher Disstress häufig mit verringerter Produktivität, höherer Arbeitnehmerfluktuation oder frühzeitiger Beren- tung assoziiert [5, 16]. Drittens wird durch disstressbedingte Erkrankungen die Behandlungsqualität der Patienten in Mitleidenschaft gezogen. Zunahme der medizinischen Behandlungsfehler, Patientenunzufriedenheit oder verringerte Medikamentenadhärenz können die Folge sein $[8,17,19]$.

Bei der Ursachensuche für das Auftreten von Disstress lassen sich entsprechend einer Übersichtsarbeit von Angerer aus dem Jahr 2008 vier Dimensionen unterscheiden: die Arbeitsbedingungen im Krankenhaus, die Diskrepanz zwischen Anforderungen und Kontrolle, berufliche Gratifikationskrisen sowie eine mangelhafte, positive Feedback- und Unterstützungskultur [1].

Zur Bewältigung dieser Ursachen von Disstress im Arztberuf und somit zur Primärprävention von disstressbedingten Erkrankungen kann das Konzept der Resilienz herangezogen werden. Die Relevanz der Resilienzförderung zur Primärprävention psychischer Erkrankungen lässt sich an der Aufnahme in die WHO-Agenda „Health 2020“ und die „sustainable development goals“ der Vereinten Nationen erkennen [21]. Der Begriff der Resilienz wurde maßgeblich durch die Kauai-Längsschnittstudie von Emmy Werner et al. aus den 1980erJahren geprägt und ist seitdem jedoch mehrfach weiterentwickelt worden. Im klassischen Sinne nach Emmy Werner ist unter individueller Resilienz kein „Charaktermerkmal“ zu verstehen, sondern „das Endprodukt von Pufferungspro- 
365 Artikel in der systematischen

Literaturrecherche gefunden:

The Cochrane Library: 9

EMBASE: 145

Medline (via PubMed): 211

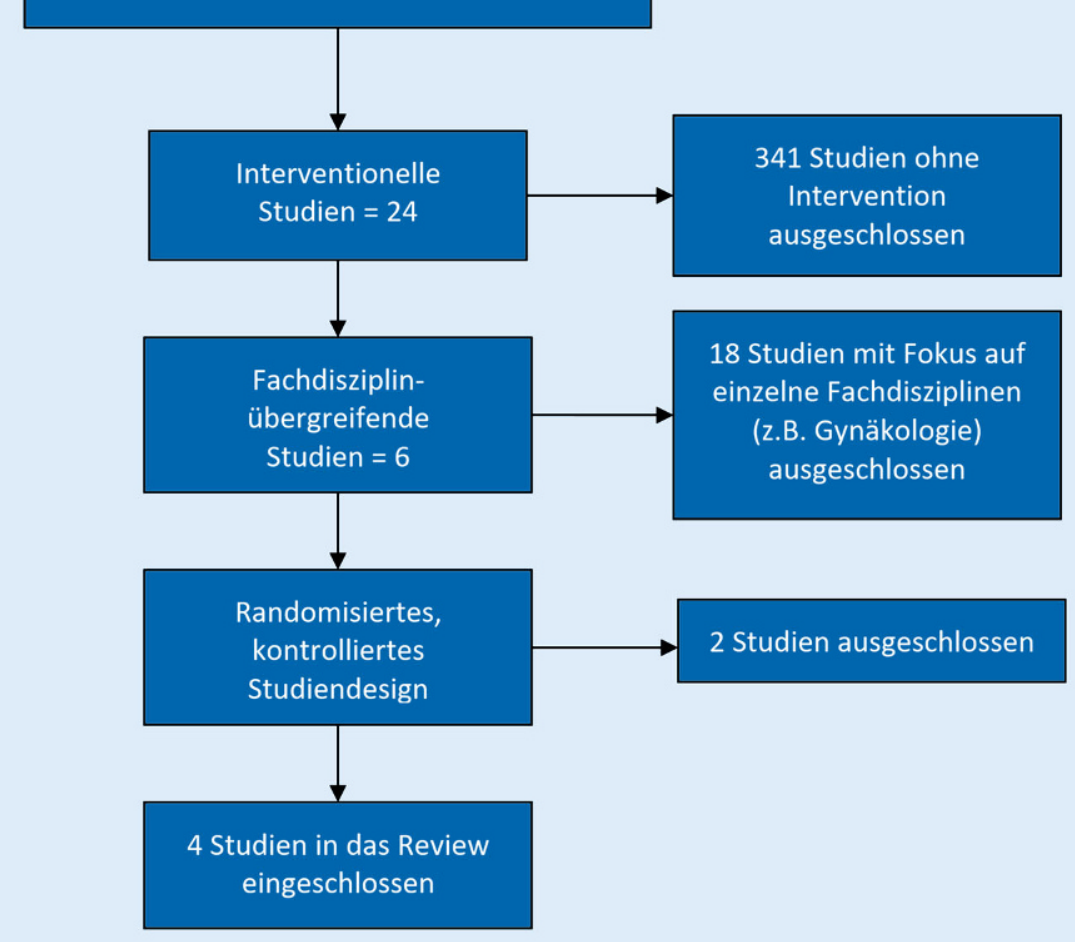

Abb. 1 A Flow-Diagramm der systematischen Literaturrecherche

zessen, welche Risiken und belastende Ereignisse zwar nicht ausschließen, es aber dem Einzelnen ermöglichen, mit ihnen erfolgreich umzugehen" [18]. Personen mit einer hohen Resilienz sind somit in der Lage, Belastungen, Bedrohungen oder Traumata erfolgreich und ohne negative physische oder psychische Folgen zu bewältigen. In einer qualitativen Studie aus dem Jahr 2011 wurden insgesamt 30 Einflussfaktoren für eine hohe Resilienz unter Ärztinnen und Ärzten identifiziert. Diese lassen sich in die drei Kategorien ,allgemeine Kraft-, Sinn- und Freudequellen" (z.B. Gratifikationen aus der Arzt-PatientenBeziehung), „konkrete Handlungen und

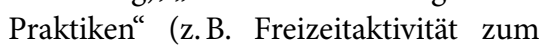
Stressabbau) und „nützliche Einstellungen und Grundhaltungen“" (z. B. Akzeptanz und Realismus) unterscheiden [22]. Der Förderung dieser Resilienzfaktoren und damit der individuellen Resilienz kann somit ein hoher Stellenwert zugeschrieben werden.

In der folgenden Arbeit soll anhand einer systematischen Literaturrecherche der Frage nachgegangen werden, inwiefern durch resilienzfördernde Interventionen der Disstress im Arztberuf reduziert werden kann.

\section{Methodik}

Entsprechend der Fragestellung wurde eine systematische Literaturrecherche in den (vom Autor als relevant erachteten) Datenbanken The Cochrane Library, Medline (via PubMed) und Embase durchgeführt. Für The Cochrane Library und Medline wurden folgende indexierten Schlagwörter (MeSH-Terms) und Suchbegriffe verwendet: „physicians", „stress“, „stress psychological“, „occupational stress", „resilience“. Da Embase über eigene indexierte Schlagwörter verfügt, wurden hierbei folgende indexierten Schlagwörter und Suchbegriffe angewandt: "physician“, „stress", „mental stress", „psychological stress", „job stress" und „resilience“. Es erfolgte eine Verknüpfung mittels der Operatoren "AND“ und „OR“. Berücksichtigt wurden nur Publikationen in englischer oder deutscher Sprache. Die systematische Literaturrecherche wurde Anfang des Jahres 2018 durchgeführt, daher wurde durch den Autor der Publikationszeitraum von 2011-2018 gewählt. Ein- und Ausschluss der gefundenen Studien erfolgte entsprechend des FlowDiagramms (• Abb. 1).

\section{Ergebnisse}

Zur Darstellung der Studienergebnisse wurde ein chronologisches Vorgehen entsprechend des Publikationsjahrs gewählt. Eine Übersicht aller Arbeiten ist in der - Tab. 1 dargestellt.

\section{Studie 1 (Sood et al.)}

Im Jahr 2011 führten Sood et al. eine randomisierte, kontrollierte Studie an der Mayo Clinic in Rochester durch. Einschlusskriterien waren die Mitgliedschaft im Departement of Medicine (DOM; 1) und die Voraussetzung, an der Intervention teilnehmen zu können und zu wollen (2). Ausschlusskriterien waren eine psychotische Episode in den letzten 6 Monaten (1) und eine andere klinisch signifikante, akute, instabile Erkrankung, die eine Teilnahme unmöglich macht (2). Die 40 eingeschlossenen Teilnehmer/-innen wurden in die Interventionsgruppe $(n=20)$ und die Kontrollgruppe $(n=20)$ randomisiert. Aus terminlichen Gründen schieden bei der Kontrollgruppe 8 Teilnehmer/-innen wieder aus $(n=12$, [14]).

Die Intervention bestand aus einer einmaligen 90-minütigen Trainingseinheit. Der Fokus wurde auf die Bewertung und Interpretation von Erfahrungen gelegt. Die Teilnehmer/-innen sollten ihre Erfahrungen weniger mit bestehenden Vorurteilen, sondern in positiverer und flexiblerer Art und Weise bewerten. Die Kontrollgruppe wurde auf eine Warteliste 
gesetzt und keiner Intervention unterzogen [14].

Für die Bewertung von Resilienz bzw. Stress wurden die „Connor-Davidson Resilience Scale“ (CD-RS) bzw. die „Perceived Stress Scale“ (PSS) herangezogen. Die Erhebung fand vor und 8 Wochen nach der Intervention statt. Im Vergleich der Interventions- zur Kontrollgruppe zeigte sich eine signifikante Zunahme der Resilienz und eine signifikante Stressreduktion nach 8 Wochen $(\mathrm{MW} \pm \mathrm{SD})$ : CD-RS $+9,8 \pm 9,6$ (Baseline: $69,6 \pm 13,3$ ) vs. $-0,8 \pm 8,2$ (Baseline: $68,0 \pm 11,2 ; p=0,003)$ und PSS $-5,4 \pm 8,1$ (Baseline: $28,2 \pm 5,9$ ) vs. $+2,2 \pm 6,1$ (Baseline: $26,2 \pm 6,9 ; p=0,010,[14])$.

\section{Studie 2 (West et al.)}

West et al. prüften 2014 die Hypothese, dass eine kleingruppenbasierte Intervention das Wohlbefinden von Ärztinnen und Ärzten verbessern kann. Wie bereits in Studie 1 wurde auch diese Studie am DOM der Mayo Clinic in Rochester durchgeführt. Insgesamt wurden 74 Ärztinnen und Ärzte à jeweils 37 Personen auf zwei Gruppen randomisiert. Sowohl in der Interventions- als auch in der Kontrollgruppe wurde den Teilnehmern/-innen alle 2 Wochen eine bezahlte Stunde „of protected time“ gewährt. Während die Teilnehmer/-innen in der Kontrollgruppe über die Ausgestaltung dieser Stunde frei verfügen konnten, nahmen die Ärzte/-innen in der Interventionsgruppe an einstündigen Treffen alle 2 Wochen für 9 Monate und damit insgesamt 19 Treffen teil [20].

Die Teilnehmer/-innen in der Interventionsgruppe wurden gleichmäßig bezüglich Geschlecht und internistischer Subspezialisierung auf 4 Kleingruppen aufgeteilt. In den Sitzungen wurden u. a. die Themen „Work-Life-Balance“, der Umgang mit medizinischen Fehlern, Gemeinschaft sowie Gender- und Generationenunterschiede bearbeitet [20].

Die Datenerhebungen fanden mittels validierter Messinstrumente in Form von Fragebögen statt. Zur Abbildung der individuell wahrgenommenen Stressbeanspruchung wurde die PSS verwendet. Resilienz wurde nicht gemessen. Die NichtTeilnehmer/-innen beantworteten einen

Präv Gesundheitsf 2020 · 15:326-331 https://doi.org/10.1007/s11553-020-00773-1

(c) Der/die Autor(en) 2020

\section{F. Kerwagen}

\section{Disstressassoziierte Erkrankungen im Arztberuf - Wie wirksam sind resilienzfördernde Interventionen?}

\author{
Zusammenfassung \\ Hintergrund. Ärztliches Personal ist starkem \\ beruflichem Disstress ausgesetzt. Im Vergleich \\ zur Normalbevölkerung besteht eine \\ erhöhte Prävalenz von disstressassoziierten \\ Erkrankungen wie Depression, Burnout und \\ Suchtverhalten. \\ Fragestellung. Können resilienzfördern- \\ de Interventionen ärztlichen Disstress \\ reduzieren? \\ Material und Methoden. Systematische \\ Literaturrecherche in den Datenbanken „The \\ Cochrane Library", "Medline“ (via "PubMed") \\ und "Embase" im Zeitraum von 2011-2018. \\ Vergleich von vier randomisiert kontrollierten \\ Studien.
}

Ergebnisse. Resilienzfördernde Interventionen können ärztlichen Disstress reduzieren. Insbesondere Berufsanfänger/-innen und weibliches ärztliches Personal scheinen davon zu profitieren.

Diskussion. Da bereits Kurzinterventionen einen positiven Effekt zeigen, sollten resilienzfördernde Maßnahmen im beruflichen Alltag von Ärztinnen und Ärzten implementiert werden.

Schlüsselwörter

Arzt · Disstress · Resilienz - Gesundheitsförderung - Übersichtsarbeit

\section{Stress-associated diseases among physicians—how effective are interventions to increase resilience?}

\section{Abstract}

Background. Among physicians there is an increased prevalence of stress-associated diseases like depression or burnout and even an increased risk of suicide and drug abuse. Objectives. Can interventions among physicians increase resilience and decrease stress?

Materials and methods. Systematic review in the Cochrane Library, MEDLINE, and Embase between 2011 and 2018. The results from four randomized controlled trials were compared. Results. Interventions can increase resilience and decrease stress among physicians.
Especially female physicians and physicians with less professional experience seem to benefit from those interventions.

Conclusions. Due to the fact that even short interventions already have a positive impact, the implementation should be possible in every hospital.

\section{Keywords}

Physicians · Stress, psychological · Resilience . Health promotion - Systematic review gekürzten Fragebogen. Neben einer Datenerhebung vor Studienbeginn fand eine Follow-up-Untersuchung 3 bzw. 12 Monate nach Studienende statt. Im Vergleich der Interventionsgruppe zur Kontrollgruppe fanden sich keine signifikanten Veränderungen bezüglich Disstress [20].

\section{Studie 3 (Mache et al.)}

In der wissenschaftlichen Literatur ist belegt, dass vornehmlich ärztliche Berufseinsteiger/-innen von den oben genannten disstressassoziierten Erkrankungen betroffen sind [12]. Aus diesem Grund entschlossen sich Mache et al. 2015, ei- ne fachdisziplinübergreifende randomisierte, kontrollierte Studie bezüglich Disstress und Resilienz an deutschen Assistenzärztinnen und -ärzten im ersten Berufsjahr durchzuführen. Einschlusskriterien waren die Beschäftigung als Krankenhausarzt/-ärztin (1), Tätigkeit in Vollzeit (2), weniger als 1 Jahr Berufserfahrung (3), freiwillige Teilnahmebereitschaft und -fähigkeit (4) und das Einverständnis, mindestens 2-mal einen Fragebogen zu beantworten (5).

Insgesamt wurden 82 Teilnehmer/ -innen auf zwei Gruppen randomisiert. Die Ärztinnen und Ärzte in der Interventionsgruppe wurden wiederum 


\begin{tabular}{|c|c|c|c|c|c|c|}
\hline Autoren & Studie & $n$ & Population & Intervention & Kontrollgruppe & Ergebnisse \\
\hline Sood et al. (2011) [14] & RCT\#1 & 32 & Physicians & $1 \times 90 \min$ & $\begin{array}{l}\text { Keine Interven- } \\
\text { tion }\end{array}$ & $\begin{array}{l}\text { Resilienz } \uparrow \\
\text { Stress } \downarrow\end{array}$ \\
\hline West et al. (2014) [20] & RCT\#2 & 74 & Physicians & $\begin{array}{l}\text { Zweiwöchentlich } 60 \text { min für } \\
9 \text { Monate }\end{array}$ & $60 \mathrm{~min}$ „Freizeit" & Stress $\leftrightarrow$ \\
\hline Mache et al. (2015) [7] & RCT\#3 & 82 & $\begin{array}{l}\text { Physicians (erstes Berufs- } \\
\text { jahr) }\end{array}$ & $\begin{array}{l}\text { Wöchentlich } 120 \text { min für } 3 \text { Mo- } \\
\text { nate }\end{array}$ & $\begin{array}{l}\text { Keine Interven- } \\
\text { tion }\end{array}$ & $\begin{array}{l}\text { Resilienz } \uparrow \\
\text { Stress } \downarrow\end{array}$ \\
\hline $\begin{array}{l}\text { Schroeder et al. (2016) } \\
\text { [10] }\end{array}$ & RCT\#4 & 33 & Physicians & $\begin{array}{l}1 \times \text { Wochenende } \\
(13 h)+2 \times 2 h\end{array}$ & $\begin{array}{l}\text { Keine Interven- } \\
\text { tion }\end{array}$ & $\begin{array}{l}\text { Resilienz } \leftrightarrow \\
\text { Stress } \downarrow\end{array}$ \\
\hline
\end{tabular}

auf 4 Kleingruppen aufgeteilt. In dieser Kleingruppengröße nahmen sie wöchentlich an einer 2-stündigen Sitzung teil. Innerhalb des Resilienztrainings wurden verschiedene Themenbereiche wie Kommunikationstechniken, das korrekte Setzen von Zielen, Motivationssteigerung oder Selbstwirksamkeit bearbeitet. Die Intervention dauerte insgesamt 12 Wochen. Die Kontrollgruppe erhielt kein Training, nahm jedoch an der Datenerhebung teil. Diese fand vor der ersten Intervention (Baseline), sowie 3 (Follow-up 1) bzw. 6 Monate (Followup 2) nach Interventionsende statt [7].

Die Datenerhebung wurde mittels standardisierter Fragebögen und Messinstrumenten durchgeführt. Zur Abbildung der individuell wahrgenommenen Stressbeanspruchung wurde der „Perceived Stress Questionnaire“ (PSQ) und zur Bestimmung der Resilienz der „Brief Resilient Coping Scale“ (BRCS) gewählt [7].

Für die Interventionsgruppe zeigte sich ein signifikanter Anstieg der Resilienz $(p=0,1)$ von $54,3 \pm 17,3$ (Baseline) auf $61,8 \pm 18,4$ (Follow-up 1) bzw. $61,5 \pm 17,9$ (Follow-up 2), während in der Kontrollgruppe die Werte nahezu gleichblieben: $53,1 \pm 16,9$ (Baseline) auf $52,8 \pm 18,4$ (Follow-up 1) bzw. 52,9 $\pm 17,8$ (Follow-up 2). Es konnte ebenfalls eine signifikante Stressreduktion $(p=0,1)$ in der Interventionsgruppe von 58,1 $\pm 19,3$ (Baseline) auf 51,8 \pm 20,7 (Follow-up 1, $p=0,1$ ) bzw. 52,3 $\pm 20,4$ (Follow-up 2, $p=0,1)$ nachgewiesen werden. In der Kontrollgruppe lagen die Werte bei $56,7 \pm 19,8$ (Baseline), 56,2 $\pm 20,9$ (Follow-up 1) und 56,4 $\pm 20,1$ (Follow-up 2; [7]).

\section{Studie 4 (Schroeder et al.)}

Um den Effekt einer kurzen Intervention auf Burnout, Stress, Achtsamkeit und Resilienz unter Ärztinnen und Ärzten zu testen, führten Schroeder et al. 2016 eine randomisierte, kontrollierte Studie am Providence Health and Services in Portland durch. Einschlusskriterien waren eine ärztliche Anstellung bei der Providence Medical Group (1), ein Beschäftigungsverhältnis von mindestens $30 \%$ (2), Alter von 25 bis 75 Jahren (3), die Bereitschaft an der Studie teilzunehmen (4) und keine bisherige Teilnahme an einer ähnlichen Intervention an der PMG (5; [10]).

Insgesamt 33 Ärztinnen und Ärzte wurden auf die Interventionsgruppe ( $n=17)$ und die Kontrollgruppe $(n=16)$ randomisiert. Die Intervention bestand aus einem 13-stündigen Trainingsprogramm am Wochenende, gefolgt von einer jeweils 2-stündigen Einheit nach weiteren 2 bzw. 4 Wochen. Im Zentrum dieser Treffen stand ein Achtsamkeitstraining mit Fokus auf den professionellen Kontext von Ärztinnen und Ärzten. Die Personen der Kontrollgruppe wurden auf eine Warteliste gesetzt und unterliefen keiner Intervention [10].

Mittels Fragebögen wurden einerseits die Teilnehmer/-innen selbst und andererseits die Patienten/-innen befragt. Die Ergebnisse wurden durch validierte Messinstrumente und Skalen ausgewertet. Zur Evaluation der Ärzteschaft bezüglich Resilienz bzw. Stress wurde die „Brief Resilience Scale“ (BRS) bzw. die „Perceived Stress Scale“ (PSS10) angewandt. Neben einer BaselineMessung vor Beginn der Intervention fand eine Follow-up-Untersuchung nach 3 Monaten statt [10].
Hinsichtlich demographischer Parameter fanden sich keine signifikanten Unterschiede zwischen den beiden Gruppen. Die Teilnehmer/-innen in der Interventionsgruppe gaben bei der Followup-Untersuchung im Vergleich zur Baseline eine signifikante Stressreduktion von $19,43 \pm 5,30$ auf $13,23 \pm 5,19(p<0,001)$ vs. $21,64 \pm 4,52$ auf $20,21 \pm 6,65$ (Kontrollgruppe). Es ließ sich keine signifikante Verbesserung der Resilienz feststellen [14].

\section{Diskussion}

Bei der systematischen Literaturrecherche wurde bewusst eine Vorgehensweise gewählt, die eine Betrachtung einzelner Ärztegruppen (z.B. Chirurgie) vermeidet, sondern der Fokus auf die Gesamtheit der Ärzteschaft gelegt. Es kann durchaus diskutiert werden, inwiefern einzelne Fachdisziplinen unterschiedliche Arbeitsbedingungen bieten bzw. Populationseigenschaften aufweisen. Um jedoch eine allgemein gültige Aussage für die gesamte Ärzteschaft treffen zu können, wurde der oben genannte Ansatz gewählt. Es liegt zudem die Vermutung nahe, dass gerade Berufsanfänger/-innen in allen Fachdisziplinen beruflichem Disstress gleichermaßen ausgesetzt sind und resilienzfördernde Maßnahmen bedürfen.

Insgesamt weisen die Ergebnisse der Studien eine Steigerung der Resilienz und eine Stressreduktion durch die jeweilige Intervention nach. Kumulativ betrachtet fanden sich lediglich in zwei von insgesamt sieben primären Outcomeparametern aller Studien keine signifikanten Unterschiede bei Stress bzw. Resilienz. Bezüglich Geschlecht und Gender lassen 
sich in allen Studien keine Unterschiede ableiten. Interessant ist der Aspekt, dass die Effekte unabhängig von der Interventionsdauer und -frequenz zu seien scheinen. Selbst die einmalige 90-minütige Intervention bei Sood et al. führte zu signifikanten Veränderungen. Die Implementation solch einer kurzen Einheit in die Praxis sollte im Alltag keine Probleme bereiten.

Der Vermutung, dass Berufsanfänger/-innen eine besonders vulnerable Population darstellen, trägt die Studie von Mache et al. Rechnung und weist einen positiven Effekt der Intervention nach. Dies deckt sich mit den Subgruppenanalysen in einer anderen Studie von Goldhagen et al. aus dem Jahr 2015. In dieser wird zudem festgestellt, dass v. a. Ärztinnen von resilienzfördernden Maßnahmen profitieren [6].

Folgende Limitationen sind $\mathrm{zu}$ berücksichtigen: Die Fallzahlen sind im Durchschnitt relativ klein und haben daher nur eine beschränkte Aussagekraft. Einschränkend muss angenommen werden, dass v. a. motivierte Ärztinnen und Ärzte an den Studien teilgenommen haben (Selektionsbias). Es bleibt unklar, inwiefern es bei den Gruppeninterventionen zu einer Beeinflussung der Ergebnisse durch ein gestärktes Gemeinschaftsgefühl bzw. soziale Unterstützung durch Gleichgesinnte kam. Der Langzeiteffekt der Interventionen wird bei einer maximalem Follow-up von $12 \mathrm{Mo-}$ naten ebenfalls nicht endgültig geklärt und bedarf längerer Studienprotokolle. Weitere Einschränkungen sind, dass in Studie 1 keine genaue Angaben zum Interventionsprotokoll und in Studie $2 \mathrm{zu}$ den Ein-/Ausschlusskriterien gemacht wurden. Eine Vergleichbarkeit der Studienergebnisse untereinander wird des Weiteren durch die Verwendung unterschiedlicher Messinstrumente erschwert. Darüber hinaus wurden 3 der 4 verglichenen Studien in den USA durchgeführt. Ob die Ergebnisse ohne Weiteres auf das deutsche Gesundheitssystem übertragen werden können, ist fraglich. Zuletzt ist darauf hinzuweisen, dass jeweils sehr unterschiedliche Interventionsinhalte angewandt wurden.

Konkret sollte eine Umsetzung in die Praxis mit einer einmaligen 90- minütigen Interventionsfrequenz im halbjährigen Turnus oder, falls möglich, ein begleitendes Modell mit einem z.B. 60-minütigen festen Gruppentermin pro Woche ubiquitär implementierbar sein. Insbesondere Berufsanfängern/ -innen sollte diese Möglichkeit angeboten werden. Mindestanforderung an jeden Arbeitgeber sollte eine Umfrage bezüglich Stress und Resilienz unter den ärztlichen Mitarbeitern sein, um das Bewusstsein für dieses Thema $\mathrm{zu}$ schärfen und dann dementsprechende Maßnahmen einleiten zu können.

\section{Fazit für die Praxis}

\section{- Disstressassoziierte Erkrankungen wie Depression und Burnout bei Ärztinnen und Ärzten sind ein Public- Health-Problem in Deutschland. \\ - Resilienzfördernde Interventionen können ärztlichen Disstress reduzie- ren. \\ - Berufsanfänger/-innen und weib- liches ärztliches Personal scheinen unabhängig von der Fachdisziplin besonders von resilienzfördernden Interventionen zu profitieren.}

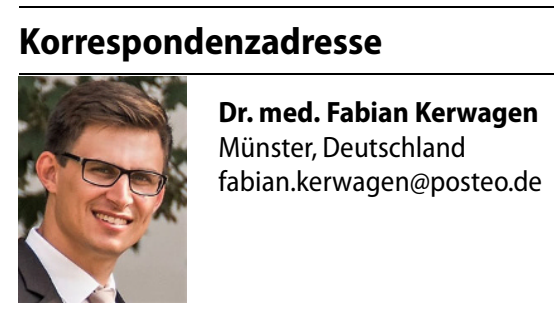

Funding. Open Access funding provided by Projekt DEAL.

\section{Einhaltung ethischer Richtlinien}

Interessenkonflikt. F. Kerwagen gibt an, dass kein Interessenkonflikt besteht.

Für diesen Beitrag wurden vom Autor keine Studien an Menschen oder Tieren durchgeführt. Für die aufgeführten Studien gelten die jeweils dort angegebenen ethischen Richtlinien.

Open Access Dieser Artikel wird unter der Creative Commons Namensnennung 4.0 International Lizenz veröffentlicht, welche die Nutzung, Vervielfältigung, Bearbeitung, Verbreitung und Wiedergabe in jeglichem Medium und Format erlaubt, sofern Sie den/die ursprünglichen Autor(en) und die Quelle ordnungsgemäß nennen, einen Link zur Creative Commons Lizenz beifügen und angeben, ob Änderungen vorgenommen wurden.

Die in diesem Artikel enthaltenen Bilder und sonstiges Drittmaterial unterliegen ebenfalls der genannten Creative Commons Lizenz, sofern sich aus der Abbildungslegende nichts anderes ergibt. Sofern das betreffende Material nicht unter der genannten Creative Commons Lizenz steht und die betreffende Handlung nicht nach gesetzlichen Vorschriften erlaubt ist, ist für die oben aufgeführten Weiterverwendungen des Materials die Einwilligung des jeweiligen Rechteinhabers einzuholen.

Weitere Details zur Lizenz entnehmen Sie bitte der Lizenzinformation auf http://creativecommons.org/ licenses/by/4.0/deed.de.

\section{Literatur}

1. Angerer P, Petru R, Nowak D et al (2008) Arbeitsbedingungen und Depression bei Ärzten. Dtsch Med Wochenschr 133(1-2):26-29

2. Angerer P, Glaser J, Petru R et al (2011) Arbeitsbedingungen der Klinikärzte: Gezielte Maßnahmen, die sich lohnen. Dtsch Arztebl 108(15):A-832

3. Assunção AA, Machado CJ, Prais HAC et al (2014) Depression among physicians working in public healthcare in Belo Horizonte, Brazil. Soc Psychiatry Psychiatr Epidemiol 49(8):1219-1229

4. Bundesärztekammer(2018) Ergebnisse derÄrztestatistik zum 31. Dezember 2018. Montgomery: Es ist höchste Zeit, den Ärztemangel ernsthaft zu bekämpfen. https://www.bundesaerztekammer.de/ ueber-uns/aerztestatistik/aerztestatistik-2018/. Zugegriffen: 28. Febr. 2020

5. Dewa CS, Loong D, Bonato S et al (2014) How does burnout affect physician productivity? A systematic literature review. BMC Health Serv Res 14:325

6. Goldhagen BE, Kingsolver $\mathrm{K}$, Stinnett $\mathrm{SS}$ et al (2015) Stress and burnout in residents: impact of mindfulness-based resilience training. Adv Med Educ Pract 6:525-532

7. Mache S, Vitzthum K, Klapp BF et al (2015) Evaluation of a multicomponent psychosocial skill training program for junior physicians in their first year at work: a pilot study. Fam Med 47(9):693-698

8. Ratanawongsa N, Roter D, Beach MC et al (2008) Physician burnout and patient-physician communication during primary care encounters. JGen Intern Med 23(10):1581-1588

9. Schernhammer ES, Colditz GA (2004) Suicide rates among physicians: a quantitative and gender assessment (meta-analysis). Am J Psychiatry 161(12):2295-2302

10. Schroeder DA, Stephens E, Colgan D et al (2018) A brief mindfulness-based intervention for primary care physicians: a pilot randomized controlled trial. Am J Lifestyle Med 12(1):83-91

11. Selye H (1973) The evolution of the stress concept: the originator of the concept traces its development from the discovery in 1936 of the alarm reaction to modern therapeutic applications of syntoxic and catatoxic hormones. Am Sci 61(6):692-699

12. Sen S, Kranzler HR, Krystal JH et al (2010) A prospective cohort study investigating factors associated with depression during medical internship. Arch Gen Psychiatry 67(6):557-565

13. Shanafelt TD, Boone $S$, Tan L et al (2012) Burnout and satisfaction with work-life balance among US 
physicians relative to the general US population. Arch Intern Med 172(18):1377-1385

14. Sood A, Prasad K, Schroeder D et al (2011) Stress management and resilience training among Department of Medicine faculty: a pilot randomized clinical trial. J Gen Intern Med 26(8):858-861

15. Tsai Y-C, Liu C-H (2012) Factors and symptoms associated with work stress and health-promoting lifestyles among hospital staff: a pilot study in Taiwan. BMCHealth Serv Res 12(1):199

16. Waldman JD, Kelly F, Arora S et al (2004) The shocking cost of turnover in health care. Health Care Manage Rev 29(1):2-7

17. Wallace JE, Lemaire JB, Ghali WA (2009) Physician wellness: a missing quality indicator. Lancet 374(9702):1714-1721

18. Werner EE (2011) Risiko und Resilienz im Leben von Kindern aus multiethnischen Familien. In: Zander M (Hrsg) Handbuch Resilienzförderung, 1. Aufl. VS, Wiesbaden, S32-46

19. West CP, Huschka MM, Novotny PJ et al (2006) Association of perceived medical errors with resident distress and empathy: a prospective longitudinal study. JAMA 296(9):1071-1078

20. West CP, Dyrbye LN, Rabatin JT et al (2014) Intervention to promote physician well-being, job satisfaction, and professionalism: a randomized clinical trial. JAMA Intern Med 174(4):527-533

21. World Health Organization (2017) Strengthening resilience: a priority shared by health 2020 and the sustainable development goals

22. ZwackJ, Schweitzer J (2011) Resilienz im Arztberuf. Universitätsklinikum Heidelberg, Heidelberg 